\title{
Higher levels of $\lg A$ and $\lg G$ at sepsis onset are associated with higher mortality: results from the Albumin Italian Outcome Sepsis (ALBIOS) trial
}

\author{
Laura Alagna ${ }^{1 \dagger}$, Jennifer M. T. A. Meessen ${ }^{2 \dagger}$, Giacomo Bellani ${ }^{3,4}$, Daniela Albiero ${ }^{3}$, Pietro Caironi ${ }^{5,6}$, \\ Irene Principale ${ }^{5}$, Luigi Vivona ${ }^{7}$, Giacomo Grasselli7, ${ }^{7}$, Francesca Motta ${ }^{2}$, Nicolò M. Agnelli ${ }^{2}$, Vieri Parrini ${ }^{9}$, \\ Stefano Romagnoli ${ }^{10,11}$, Roberto Keim ${ }^{12}$, Francesca Di Marzo Capozzi ${ }^{13}$, Fabio S. Taccone ${ }^{14}$, Walter Taccone ${ }^{13}$, \\ Barbara Bottazzi ${ }^{15}$, Alessandra Bandera ${ }^{1,7}$, Andrea Cortegiani ${ }^{16,17}$ and Roberto Latini ${ }^{2^{*}}$ (i)
}

\begin{abstract}
Background: The role of intravenous immunoglobulins (IVIG) during sepsis is controversial, as different trials on IVIG have observed inconsistent survival benefits. We aimed to elucidate the possible association and clinical significance between circulating levels of immunoglobulins.

Methods: In a subset of 956 patients with severe sepsis and septic shock of the multicentre, open-label RCT ALBIOS, venous blood samples were serially collected 1, 2, and 7 days after enrolment (or at ICU discharge, whichever came first). IgA, IgG and IgM concentrations were assayed in all patients on day 1 and in a subgroup of 150 patients on days 2 and 7. Ig concentrations were measured employing a turbidimetric assay, OSR61171 system.

Results: IgA on day 1 had a significant predictive value for both 28-day and 90-day mortality (28-day mortality, HR: 1.50 (95\% Cl 1.18-1.92); 90-day mortality, HR: 1.54 (95\% Cl 1.25-1.91)). lgG, but not lgM, on day 1 showed similar results for 28-day (HR 1.83 (95\% Cl 1.33-2.51) and 90-day mortality HR: 1.66 (95\% Cl 1.23-2.25)). In addition, lower levels of $\operatorname{lgG}$ but not of $\lg A$ and $\operatorname{lgM}$, at day 1 were associated with significantly higher risk of secondary infections (533 [406-772] vs 600 [452-842] mg/dL, median [Q1-Q3], $p=0.007$ ).

Conclusions: In the largest cohort study of patients with severe sepsis or septic shock, we found that high levels of $\lg A$ and $\lg G$ on the first day of diagnosis were associated with a decreased 90-day survival. No association was found between IgM levels and survival. As such, the assessment of endogenous immunoglobulins could be a useful tool to identify septic patients at high risk of mortality.
\end{abstract}

Trial registration \#NCT00707122, Clinicaltrial.gov, registered 30 June 2008

Keywords: IgA, IgG, IgM Immunoglobulins, Mortality, Septic shock, Sepsis

*Correspondence: roberto.latini@marionegri.it

${ }^{\dagger}$ Laura Alagna and Jennifer M.T.A. Meessen shared first authorship

2 Department of Cardiovascular Medicine, Institute for Pharmacological

Research Mario Negri IRCCS, Via Mario Negri 2, 20156 Milan, Italy

Full list of author information is available at the end of the article

\section{Background}

Sepsis is one of the main causes of admission to intensive care unit (ICU) [1]. Both sepsis and septic shock are associated with an increased risk of death, with septic shock showing a mortality rate of more than 50\% [2]. Cornerstones of the early-stage management of sepsis 
and septic shock are early recognition and resuscitation, source of infection control, adequate antibiotic therapy, achieving hemodynamic stability, and support of failing organs [3]. However, these measures and clinical therapies do not counter the inflammatory imbalance and the immunological dysregulation, including the subsequent prolonged immunosuppression, very often characterizing sepsis and septic shock $[4,5]$.

In patients suffering from these conditions, plasma concentration of all immunoglobulin classes has been reported as reduced [6, 7]. Moreover, low immunoglobulin levels during sepsis have been associated with poor outcome, suggesting the hypothesis that endogenous immunoglobulin replacement during sepsis may improve survival $[8,9]$.

The potential benefits of the use of intravenous immunoglobulins (IVIG) in severe infections is based on the two main functions of such therapy: antimicrobial activity and immunomodulatory effect [10]. Despite this rationale, the role of IVIG during sepsis is still controversial, as different randomized controlled trials (RCTs) have observed inconsistent survival benefits [11]. Moreover, even though low IgG has been reported, on average, in $58 \%$ of heterogeneous cohorts of patients on the day of diagnosis of sepsis, did not appear to be related to an increased risk of death [7]. Indeed, the nadir of immunoglobulin plasma concentration is often seen on day 3 following sepsis diagnosis [7, 12]. Moreover, other immunoglobulin subclasses (IgG1, IgM and IgA) have important immunomodulatory function and might also influence patients' outcome. Finally, the association between low immunoglobulin concentrations and mortality is often observed in septic patients with less severe organ dysfunction [13], while the prognostic role of immunoglobulins remains unclear in most severe cases. These issues, therefore, suggest that our understanding of the mechanisms underlying the high prevalence of low immunoglobulins in sepsis and their impact on severity of the disease is incomplete.

We, therefore, aimed, in the present study, to elucidate the possible association and clinical significance between circulating levels of immunoglobulins, clinical characteristics and outcomes in a large cohort of critically ill patients with severe sepsis and septic shock.

\section{Methods}

This was a secondary analysis of the Albumin Italian Outcome Sepsis (ALBIOS) trial. ALBIOS study was a multicentre, open-label, randomized, controlled trial on 1818 patients with severe sepsis or septic shock admitted to 100 Italian intensive care units (ICUs) to investigate the potential efficacy of albumin replacement (NCT00707122 clinicaltrial.gov). The study complied with the 1975 Declaration of Helsinki as revised in 2008 and was approved by the institutional review boards of each centre. Written informed consent or deferred consent was obtained from each participant, according to Italian legislation. Patients were randomly assigned to receive either $20 \%$ albumin and crystalloids or crystalloids alone for fluid resuscitation, from randomization until day 28 or discharge from the ICU, whichever came first. Primary outcome was 90 day mortality. Albumin administration was targeted to achieve a serum concentration of $30 \mathrm{~g} / \mathrm{L}$ or more. Study design, inclusion and exclusion criteria, and main results have been published elsewhere [14].

\section{Definitions}

In the original ALBIOS trial and for the purpose of this analysis, severe sepsis was defined as a proved or suspected infection in at least one site, two or more signs of systemic inflammatory reaction, and at least one severe, acute sepsis-related organ dysfunction [15]. Organ function was assessed on a daily basis with the use of the Sequential Organ Failure Assessment (SOFA) score [16], which rates for each of five organ sub-components (respiratory, coagulation, liver, cardiovascular and renal systems) from 0 to 4 , with higher scores indicating a greater degree of organ dysfunction. Septic shock at the time of randomization was defined as severe sepsis with a SOFA score of $\geq 3$ of the cardiovascular subcomponent.

\section{Assays}

In a subset of 956 patients recruited in 40 centres that participated in a predefined biologic sub-study, venous blood samples were serially collected 1,2 , and 7 days after enrolment (or at ICU discharge, whichever came first). Blood samples were collected into ethylenediaminetetraacetic acid (EDTA) tubes, centrifuged, plasma stored at $-70{ }^{\circ} \mathrm{C}$, and shipped on dry ice to a central repository at $-70{ }^{\circ} \mathrm{C}$ until assayed. All assays were performed by expert personnel, blinded to identity of plasma samples. Total immunoglobulin concentrations (IgG, IgA, and IgM) were assayed in all patients on day 1 and in a subgroup of 150 patients also on day 2 and 7. Ig concentrations were measured at Futura Diagnostica srl (Avellino, Italy) employing a turbidimetric assay, OSR61171 system (Beckman Coulter Inc., 250 S. Kraemer Blvd. Brea, CA 92821, USA). Calibration for the immunoglobulin procedure was performed every 90 days, using specific calibrators. Two levels of an appropriate immunology control material were tested daily as quality control, in accordance with regulatory requirements and laboratory standard procedure. The Limit of Quantitation (LOQ) for the immunoglobulin assay was $10 \mathrm{mg} / \mathrm{dL}$. 
In addition, data on inflammation biomarkers such as Presepsin [17], Pentraxin3 (PTX3) [18], Pro-protein convertase subtilisin/kexin 9 (PCSK9) [19], Resistin, myeloperoxidase (MPO) [20] and osteopontin (OPN) [21], already assayed in ALBIOS was included in this manuscript.

\section{Statistical analysis}

Patients were divided into subgroups based on the levels of IgA, IgG and IgM on day 1 as follows [22-25]:

- "LOW" (i.e., IgA: $>70 \mathrm{mg} / \mathrm{dL}, \operatorname{IgG}:<700 \mathrm{mg} / \mathrm{dL}$ and IgM: $<40 \mathrm{mg} / \mathrm{dL})$;

- "NORMAL" (i.e., IgA: 70-400 mg/dL, IgG: 700$1600 \mathrm{mg} / \mathrm{dL}$ and IgM: 40-230 mg/dL);

- "HIGH" (i.e., IgA: > 400 mg/dL, IgG > 1600 mg/dL and $\operatorname{IgM}>230 \mathrm{mg} / \mathrm{dL})$.

Patient characteristics were compared across these groups by means of Chi-square or Kruskal-Wallis test, as appropriate. Those variables found to be significantly different between these Ig-groups are then included in continuous multivariable linear regression models to assess which variables were independently associated with Iglevels. Time course of immunoglobulins at day 1, 2 and 7 was assessed by means of repeated measures analysis, including a Greenhouse-Geisser correction if Mauchley's test for sphericity was violated.

Kaplan-Meier curves were constructed to assess the association of the three Ig-groups and survival. Restricted cubic splines including three knots were created for 90-day mortality. Based upon these curves, only the values above the inflection point of the curve were included in multivariable survival analysis, relating z-transformed immunoglobulins to 28 - and 90-day mortality by means of Cox proportional hazards models. These regression analyses were adjusted for the variables found to be independently associated with 28 - and 90-mortality with a $p<0.0001$. Association of Ig-levels and secondary infections was assessed by Kruskal-Wallis test. Data were analysed by means of SPSS Version 25 (IBM SPSS, Armonk, $\mathrm{NY}$ ) and R-package "rms" ( $\mathrm{R}$ foundation for statistical computing, Vienna, Austria).

\section{Results}

\section{Study population}

Concentrations on Day 1 of IgA, IgG and IgM were available for 956 patients. Demographic and clinical characteristics of the study population as divided according to immunoglobulin levels on day 1 are presented in Additional file 1: Tables S1, S2 and S3 for the total population. Characteristics of the 956 patients measured on day 1 after randomization are comparable with those of the total ALBIOS cohort are similar to those of the overall population [26]. More than half of the patients were in septic shock (56.3\%), 90-day mortality was $38.6 \%$. When stratifying patients according to immunoglobulin levels (i.e., low, normal and high), patients with the highest IgA, IgG or IgM had higher white blood cell count. Immunocompromised patients were more prevalent in the low range of IgA and in the high range of IgG. Prevalence of septic shock did not differ between ranges of IgA, $\mathrm{G}$ or $\mathrm{M}$. Mortality was higher in the high range of IgA and IgG, but no difference was found for IgM. In 150 patients, immunoglobulin levels at day 2 and day 7 were also measured: the concentrations of all three immunoglobulins significantly increased over time (over time IgA $p=1.0 \times 10^{-18} ; \operatorname{IgG} p=8.2 \times 10^{-10} ; \operatorname{IgM} p=4.3 \times 10^{-10}$ Additional file 1: Table S4). In the absence of formal statistical significance, concentrations of IgA and IgG, but not of IgM are slightly lower in albumin group at all three timepoints (Additional file 1: Table S4). A stronger correlation between IgA and IgG (Spearman $r$ : 0.595) was observed as compared to IgG ad IgM (Spearman $r$ : 0.484) and IgM and IgA (Spearman $r$ : 0.320).

\section{IgA levels}

On day 1, 85 out of 955 (8.9\%) patients had LOW (median 49, IQR [34-59] mg/dL), 824 (86.3\%) NORMAL (168.5 [120.3-221] $\mathrm{mg} / \mathrm{dL})$ and $46(4.8 \%) \mathrm{HIGH}$ circulating IgA levels (469, [443-527] mg/dL). Patients in the HIGH IgA subgroup were more frequently male and received more frequently albumin administration than others. None of the included inflammatory biomarkers was associated with IgA levels (Additional file 1: Table S1). Among comorbidities, the presence of previous immunosuppression and onco-hematologic disease was more often observed in the LOW IgA subgroup. In multivariable linear regression analyses, male sex, higher BMI, higher white blood cell count, cardiovascular comorbidities and study treatment allocation to crystalloids were all independently associated with higher IgA levels on day 1, whereas the presence of onco-hematologic pathologies was associated with lower IgA (Table 1).

\section{IgG levels}

On day 1, 608 out of 956 (63.6\%) patients had LOW (577 [372-572] $\mathrm{mg} / \mathrm{dL}), 321$ (33.6\%) had NORMAL (900 [776-1076] mg/dL) and 27 (2.8\%) HIGH IgG levels (1769 [1661-2003] mg/dL; Additional file 1: Table S2). Patients with HIGH IgG had more frequently a pulmonary infection than other groups. In the NORMAL subgroup, patients received less frequently albumin administration and had higher white blood cells count than other groups. In the LOW subgroup, patients were more frequently male, suffered more often from septic shock and 
Table 1 Multivariate linear regression analysis output for variables independently associated with $\lg \mathrm{A}$, $\lg \mathrm{G}$ and $\lg \mathrm{M}$ on day 1

\begin{tabular}{|c|c|c|c|}
\hline Variable & B & $95 \% \mathrm{Cl}$ & $p$ \\
\hline \multicolumn{4}{|l|}{$\lg A$} \\
\hline Female sex & -34.1 & $-47.5:-20.7$ & $7.0 \times 10^{-7}$ \\
\hline BMl & 2.6 & $1.4: 3.8$ & $1.5 \times 10^{-5}$ \\
\hline $\begin{array}{l}\text { White blood cell count at } \\
\text { baseline }\end{array}$ & 1.4 & $0.7: 2.1$ & $6.0 \times 10^{-5}$ \\
\hline CV comorbidities & 28.6 & 11.2: 46.1 & 0.001 \\
\hline Randomized to crystalloids & 19.4 & $6.3: 32.5$ & 0.004 \\
\hline Onco-haematologic disease & -48.0 & $-87.0:-9.1$ & 0.016 \\
\hline Creatinine & -3.5 & $-7.6: 0.6$ & 0.090 \\
\hline Immunocompromised state & -18.3 & $-40.0: 3.3$ & 0.097 \\
\hline \multicolumn{4}{|l|}{$\lg G$} \\
\hline Female sex & -88.5 & $-135.5:-41.4$ & $2.4 \times 10^{-4}$ \\
\hline $\begin{array}{l}\text { White blood cell count at } \\
\text { baseline }\end{array}$ & 4.2 & $1.7: 6.6$ & 0.001 \\
\hline Randomized to crystalloids & 73.7 & 27.5: 120.0 & 0.002 \\
\hline BMI & 5.8 & 1.7: 10.0 & 0.006 \\
\hline Shock & -55.1 & $-101.8:-8.4$ & 0.021 \\
\hline Liver disease & 188.5 & $-3.5: 380.4$ & 0.054 \\
\hline Heart rate & -0.9 & $-2.0: 0.3$ & 0.127 \\
\hline Immunocompromised state & -9.3 & $-79.1: 60.6$ & 0.794 \\
\hline \multicolumn{4}{|l|}{$\lg M$} \\
\hline Age & -0.6 & $-0.9:-0.2$ & 0.002 \\
\hline Shock & -14.4 & $-25.3:-3.6$ & 0.009 \\
\hline Onco-haematologic disease & -30.1 & $-58.2:-2.0$ & 0.036 \\
\hline $\begin{array}{l}\text { White blood cell count at } \\
\text { baseline }\end{array}$ & 0.4 & $-0.1: 1.0$ & 0.127 \\
\hline Mean arterial pressure & 0.2 & $-0.2: 0.5$ & 0.329 \\
\hline
\end{tabular}

Output for multivariable linear regression analysis with continuous Ig concentration as dependent variable

Included in the regression analyses are the variables which were significant in Additional file 1: Tables S1, S2 and S3. For IgG, AIDS was not included due to confounding by immuno-compromised state. Location of infection was not included in this analysis

presented more frequently immunodepression. PTX3 was significantly, inversely associated with IgG levels: it was highest at low levels of IgG. In the multivariable linear regression analysis, male sex, higher white blood cell count, study treatment allocation to crystalloids, higher BMI and shock were found to be independently associated with high IgG levels on day 1, whereas patients with shock as compared to patients with sepsis had lower IgG levels (Table 1).

\section{IgM levels}

On day 1, 219 out of 956 (22.9\%) patients had LOW (27 [1-33] mg/dL); 697 (72.9\%) NORMAL (78 [56115] $\mathrm{mg} / \mathrm{dL}$ ) and 40 (4.2\%) HIGH IgM levels (337
[268-434] mg/dL; Additional file 1: Table S3). In the NORMAL subgroup, patients were younger. In the LOW subgroup, patients had more frequently septic shock and low mean arterial pressure, as well as lower white blood cell count as compared to the other groups. In addition, an inverse correlation was found for PTX3 and IgM. In the multivariable linear regression analysis, higher age, the presence of septic shock and onco-hematologic disease were found to be independently associated with decreased IgM levels on day 1 (Table 1).

\section{Immunoglobulin and survival}

Patients with HIGH IgA had higher mortality (28-day mortality: $50.0 \%$; 90 -day mortality $60.9 \%$ ) as compared to the other subgroups. Similar results were also observed for the HIGH IgG subgroup. In particular, for both IgA and IgG, 28-day mortality in the HIGH group was twofold higher as compared to LOW and NORMAL ranges (Additional file 1: Tables S1 and S2). No association between IgM and mortality at any time was found. These findings were confirmed by the restricted cubic splines (Fig. 1) and Kaplan-Meier curves analyses (Fig. 2).

The restricted cubic splines showed an inflection point for mortality at $150 \mathrm{mg} / \mathrm{dL}$ for IgA and $750 \mathrm{mg} /$ $\mathrm{dL}$ for IgG, below these values we did not see any significant effect. Therefore, Cox proportional hazard survival analyses for $z$-transformed concentrations of IgA and IgG were performed only for patients above this cutoff (Table 2). Additional file 1: Table S5 depicts the baseline characteristics for survivors and non-survivors separately. Based upon this table the selection of variables to be included in multivariable regression was made: age, shock, baseline SOFA-score, creatinine, platelets, cardiovascular comorbidities, renal disease and COPD were included. IgA on day 1 had a significant predictive value for both 28-day and 90-day mortality (28-day mortality, HR: 1.50 (95\% CI 1.18-1.92), $p=0.001$ and 90-day mortality HR: 1.54 (95\% CI 1.25-1.91), $p<0.001)$. IgG concentrations on day 1 showed similar results for 28-day (HR: 1.83 (95\% CI 1.33-2.51), $p<0.001$ ) and 90-day mortality (HR: 1.66 (95\% CI 1.23-2.25), $p=0.001)$.

\section{Immunoglobulins and secondary infections}

Of the 956 patients included in this study, 246 experienced a secondary infection. Lower levels of IgG at day 1 were associated with higher risk of secondary infections (median 533 IQR 406-772 vs median 600 IQR 452-842, $p=0.007$ ). This difference was not observed for IgA and IgM. 

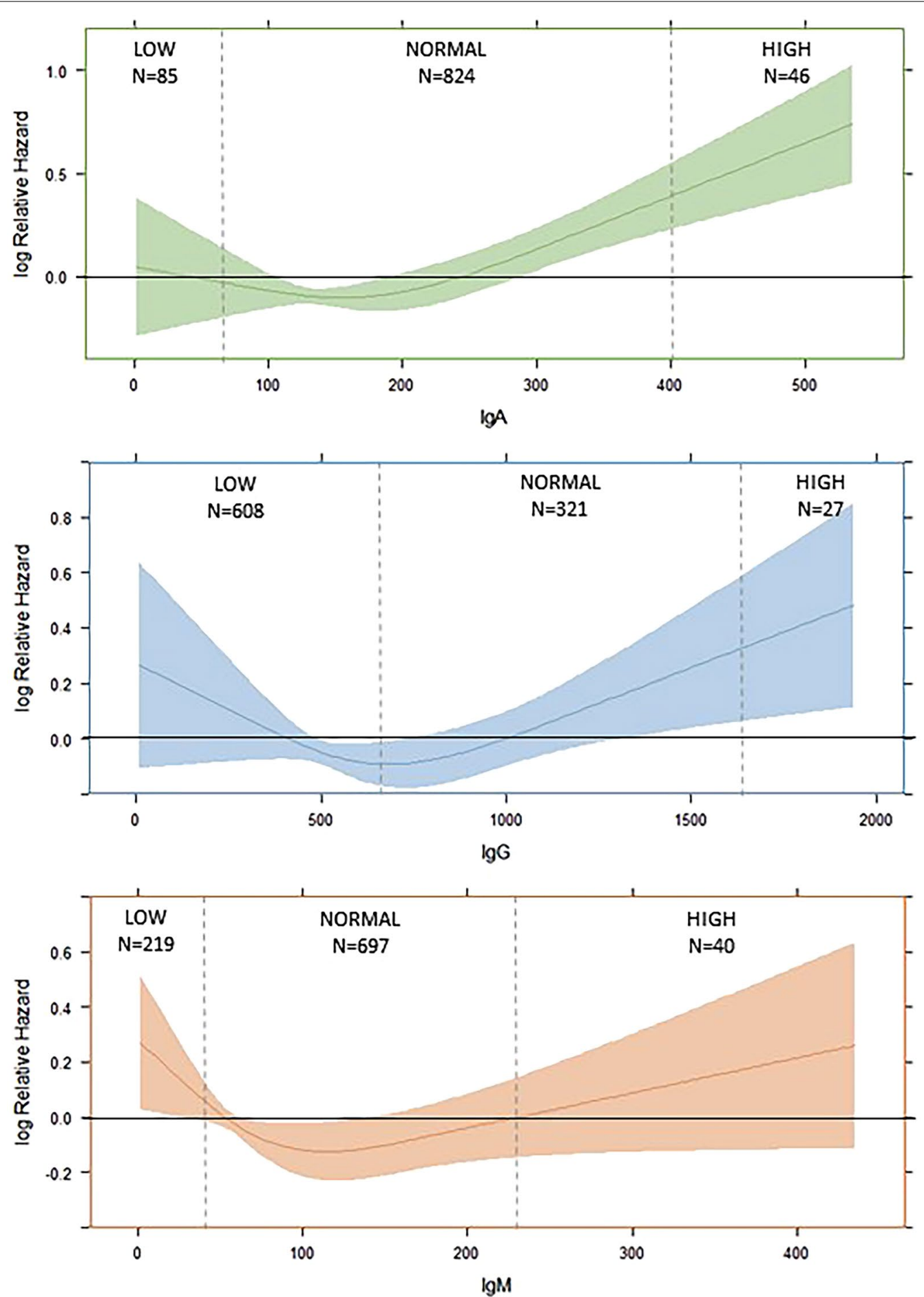

Fig. 1 Splines for 90-day mortality, dotted line represents the inflection point

\section{Discussion}

In the largest cohort study of patients with severe sepsis or septic shock, we found that high levels of IgA and IgG on the first day of diagnosis were associated with a decreased 90-day survival. No association was found between IgM levels and survival. As such, the assessment of endogenous immunoglobulins could be a useful tool to identify septic patients at high risk of mortality. 


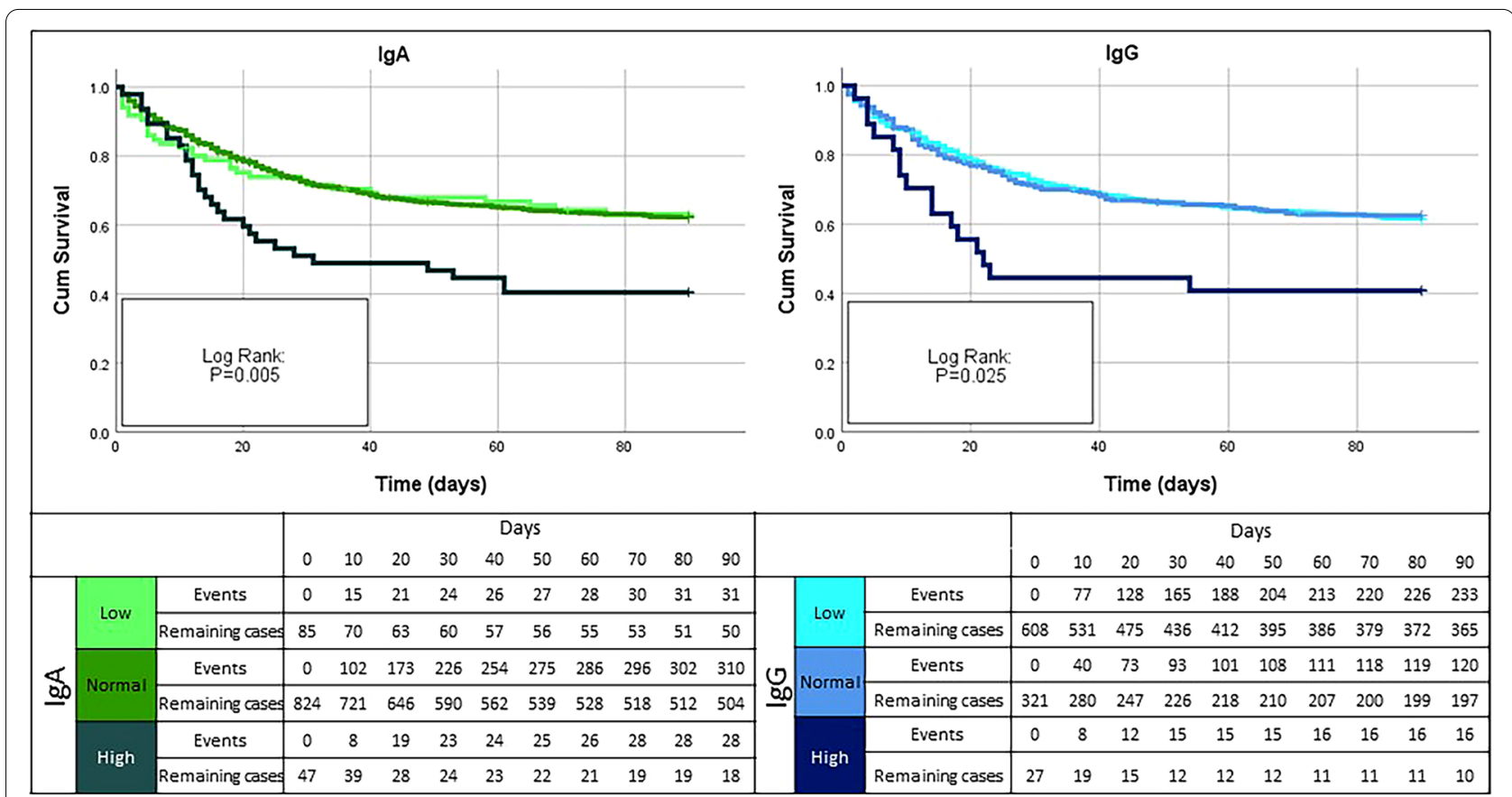

Fig. 2 Kaplan-Meier analysis for lgA and lgG-groups and 90-day mortality

Table 2 Cox regression analysis for 28- and 90-day survival

\begin{tabular}{llllll}
\hline Ig & Outcome & Model & HR & $95 \%$ Cl & P \\
\hline IgA & 28-day mortality & Univariate & $\mathbf{1 . 2 4}$ & $\mathbf{1 . 0 7 - 1 . 4 3}$ & $\mathbf{0 . 0 0 4}$ \\
& (>150 mg/dL) & Multivariate & $\mathbf{1 . 5 0}$ & $\mathbf{1 . 1 8 - 1 . 9 2}$ & $\mathbf{0 . 0 0 1}$ \\
& 90-day mortality & Univariate & $\mathbf{1 . 2 4}$ & $\mathbf{1 . 0 9 - 1 . 4 1}$ & $\mathbf{0 . 0 0 1}$ \\
& (>150 mg/dL) & Multivariate & $\mathbf{1 . 5 4}$ & $\mathbf{1 . 2 5}-\mathbf{1 . 9 1}$ & $\mathbf{6 . 3} \times \mathbf{1 0 ^ { - 5 }}$ \\
IgG & 28-day mortality & Univariate & $\mathbf{1 . 2 1}$ & $\mathbf{1 . 0 2 - 1 . 4 4}$ & $\mathbf{0 . 0 3 3}$ \\
& (>750 mg/dL) & Multivariate & $\mathbf{1 . 8 3}$ & $\mathbf{1 . 3 3 - 2 . 5 1}$ & $\mathbf{1 . 8 \times 1 0 ^ { - 4 }}$ \\
& 90-day mortality & Univariate & 1.16 & $0.99-1.37$ & 0.070 \\
& $(>750 \mathrm{mg} / \mathrm{dL})$ & Multivariate & $\mathbf{1 . 6 6}$ & $\mathbf{1 . 2 3 - 2 . 2 5}$ & $\mathbf{0 . 0 0 1}$
\end{tabular}

Based upon the restricted cubic spline for $\lg A>150$ was included $(N=124)$ and for $\lg G>750(N=294)$

All values were $Z$-standardized-HR for $1 \mathrm{SD}$

Both multivariate Cox proportional hazards regression analyses were adjusted for clinical characteristics which were associated with 28- and 90-day mortality with a $p$ value of $<0.0001$ : age, shock, baseline SOFA score, creatinine, platelets, cardiovascular comorbidities, renal comorbidities and COPD, see Additional file 1: Table S5

The three classes of immunoglobulins act synergically in the immune response. IgG mainly controls secondary antibody responses, opsonization and complement activation, IgA works for the mucosal immunity, while the key functions of IgM are complement activation and primary antibody responses [27]. The association of immunoglobulin levels with patients' survival is controversial [28]. Even if the majority of previous studies included a small number of patients, most of them reported that hypogammaglobulinemia was associated with a worse outcome [6, 7, 27]. In particular, Bermejo-Martin et al. [27] showed in 172 adult patients with severe sepsis or septic shock that low plasma concentrations of IgA, IgG and IgM were associated with reduced survival, which was not the case in ALBIOS. Those studies showed a correlation between low levels of IgG and mortality [29], but also with other clinical outcomes, such as vasopressorfree days and acute lung injury [6]. Differently from these findings, a recent meta-analysis, showed that the prevalence of hypogammaglobulinemia on the day of sepsis diagnosis is as high as $70 \%$, but no specific sub-group of patients with a higher risk of death was identified [13]. Indeed, only in patients with total SOFA score $<8$, the simultaneous presence of low levels of IgA, IgG and IgM was associated with higher mortality. More recently, results from the SBITs (Score-based immunoglobulin G therapy of patients with sepsis) study demonstrated that baseline hypogammaglobulinemia does not correlate with the survival in 543 patients with severe sepsis and septic shock. Moreover, patients with the highest IgG levels (fourth quartile, IgG $>1200 \mathrm{mg} / \mathrm{dl}$ ) had a higher mortality compared to the reference quartile $[22,30]$.

In the cohort of the ALBIOS study, higher levels of IgA and IgG were associated with a higher risk of mortality. It is known that immunoglobulins play a crucial role in 
host response to infection, given their ability to neutralize endotoxins [31], to limit cytokines production [32], to increase serum bactericidal activity [33] and to attenuate the hyper activation of the complement cascade [34]. However, it is important also to consider a paradoxical effect of immune system activation. Higher levels of immunoglobulins could be a consequence of a hyper activation of the inflammatory response that can contribute to the paralysis of immune system during this critical state [30]. In addition, the association between IgA/ IgG and mortality is independent of antibiotic therapy. In other words, the association with increased mortality of HIGH levels of IgA and IgG, cannot be explained by a different rate of appropriate antibiotic therapy.

In our study, IgM levels did not correlate with inhospital mortality, although low IgM levels were associated with the occurrence of septic shock. In a previous study, Giamarellos-Bourbolis et al. found a correlation between IgM levels and mortality, highlighting that the concentration of IgM over time was greater in survivors than in non-survivors from septic shock [35]. Data from animal and in vitro studies suggest that IgM are released by a particular class of B lymphocytes belonging to the innate defence system, containing IgM in their cytoplasmic stores. In experimental sepsis models, depletion of B-lymphocytes leads to early death [36]. Moreover, patients with sepsis produced much less IgM than healthy volunteers [35-37]. Therefore, one could argue that during sepsis B lymphocytes may produce less IgM, so that patients' necessity of adaptive immunity are compensated by circulating IgM. Once septic shock develops, circulating IgM are rapidly depleted and deficient production by B-lymphocytes cannot compensate for loss.

Recently, the analysis of clinical data from more than 20,000 patients, classified according to Sepsis-3 definition [1], allowed to define four phenotypes with different clinical characteristics [38]. Consistent differences in biomarker patterns and mortality rate were observed in the different phenotypes, and simulations suggested that these phenotypes may help understanding heterogeneity in the response to treatments. Four different genotypes were also identified by genome-wide gene expression profiles of blood from sepsis patients on admission to Intensive Care Unit for sepsis [39]. In addition, in this case, the four genotypes were characterized by different mortality rate and biomarkers profile, providing a tool for personalized patient management and novel criteria for selection of patients for trials. Therefore, the discrepancy observed when investigating the role of immunoglobulins in patient stratification and severity of the disease could be likely due to the heterogeneity of the population analysed without further classification. None of the included inflammatory biomarkers was associated with immunoglobulin levels, with the only exception of a significant inverse correlation of PTX3 with IgG and IgM levels and an association of PCSK9 to IgG. The specific inverse correlation of PTX3 with all 3 Igs, in the absence of any correlation of other inflammatory markers suggests an involvement of innate immunity, to which PTX3 pertains. The further classification of sepsis patients according to the genotypes defined by genome-wide gene expression profiles could possibly help to better understand the role of inflammatory molecules.

Several limitations are worth mentioning. First, we did not assay specific subtypes of immunoglobulins, which may have provided useful clues to the interpretation of the data. Second, we did not assess the time course of Ig's in the whole cohort of patients, due to limitations in resources. Third, it was not possible to investigate the relation between the etiological agent of sepsis, where present, and the immunoglobulin time course.

In our study low baseline levels of IgG correlate with the risk of secondary infections after the first sepsis episodes. To our knowledge, this is a novel finding not previously reported. It is conceivable that during the acute phase of sepsis a "paralysis" of the immune system often occurs, in addition to the consumption and loss of immunoglobulins. A poor immunoglobulin reserve can result in a lack of immune control during the late or recovery sepsis phase, allowing some other infectious episodes.

\section{Conclusion}

In this study, higher levels of IgG and IgA were associated with 90-day mortality in patients with sepsis or septic shock. Our data also suggest that the knowledge on pathophysiological mechanisms underlying the interaction of immunoglobulins during sepsis and its association with clinical outcomes are incomplete.

\section{Abbreviations \\ AIFA: Italian medicine agency; ALBIOS: Albumin Italian Outcome Septic Shock; BMI: Body Mass Index; CI 95\%: 95\% Confidence interval; EDTA: Ethylenediami- netetraacetic acid; HR: Hazard ratio; ICU: Intensive Care Unit; IgA: Immuno- globulin A; IgG: Immunoglobulin G; IgM: Immunoglobulin M; IQR: Interquartile range; IVIG: Intravenous immunoglobulins; LOQ: Limit of Quantitation; RCT: Randomized controlled trial; SBIT: Score-based immunoglobulin G therapy of patients with sepsis; SOFA: Sequential Organ Failure Assessment.}

\section{Supplementary Information}

The online version contains supplementary material available at https://doi. org/10.1186/s13613-021-00952-z.

Additional file 1: Table S1. Patient characteristics by lgA groups. Table S2. Patient characteristics by lgG groups. Table S3. Patient characteristics by lgM groups.Table S4. Plasma concentrations of IgA, IgG \& IgM on day $1,2 \& 7$ by day 1 levels. Table $\mathbf{S 5}$. Patient characteristics by 90 -day survival. 


\section{Acknowledgements}

Professor Luciano Gattinoni, Principal Investigator of ALBIOS study, supervised and inspired biologic/mechanistic sub-studies of the main trial, including the present one.

\section{Authors' contributions}

LA \& JMTAM: conception and design, analysis and interpretation of data; drafting the article. GB, DA, IP, LV, GG, FM, NMA, VP, SR, RK, FDMC, FST \&WT: acquisition of data, revising the article critically for important intellectual content. PC: conception and design, revising the article critically for important intellectual content. AB: conception and design, drafting the article. AC: acquisition of data, revising the article critically for important intellectual content. RL: conception and design, analysis and interpretation of data; revising the article critically for important intellectual content. All authors gave final approval of the version to be published and agreement to be accountable for all aspects of the work thereby ensuring that questions related to the accuracy or integrity of any part of the work are appropriately investigated and resolved. All authors read and approved the final manuscript.

\section{Funding}

This work was supported by the following grants: the ALBIOS trial was funded by a grant from the Italian Medicines Agency (AIFA, Grant FARM6JS3R5, 2006) and the Italian Ministry of Health (Ricerca Finalizzata 2011-2012, Grant RF-2011-02348358), and from Grifols (ALBUS Grifols Award, 2015). The assays of immunoglobulins for the present sub-study were performed free of charge by Futura Diagnostica srl (Avellino, Italy).

\section{Availability of data and materials}

Data supporting this study and the ALBIOS study are not publicly available.

\section{Declarations}

\section{Ethics approval and consent to participate}

The study protocol and the informed consent process were approved by the ethics committee at each participating institution. Written informed consent or deferred consent was obtained from each patient.

\section{Consent for publication}

Not applicable.

\section{Competing interests}

The authors declare that they have no competing interests.

\section{Author details}

'Department of Infectious Diseases, Fondazione IRCCS Ca' Granda Ospedale Maggiore Policlinico, Milan, Italy. ${ }^{2}$ Department of Cardiovascular Medicine, Institute for Pharmacological Research Mario Negri IRCCS, Via Mario Negri 2, 20156 Milan, Italy. ${ }^{3}$ Department of Emergency and Intensive Care, San Gerardo Hospital, Via Giambattista Pergolesi 33, 20900 Monza, MB, Italy. ${ }^{4}$ Department of Medicine and Surgery, University of Milan-Bicocca, Via Cadore 48, 20900 Monza, MB, Italy. ${ }^{5}$ Department of Anesthesia and Critical Care, AOU S. Luigi Gonzaga, Orbassano, Italy. ${ }^{6}$ Department of Oncology, University of Turin, Turin, Italy. ${ }^{7}$ Department of Pathophysiology and Transplantation, Università Degli Studi di Milano, Milan, Italy. ${ }^{8}$ Department of Anesthesia, Intensive Care and Emergency, Fondazione IRCCS Ca' Granda Ospedale Maggiore Policlinico, Milan, Italy. ${ }^{9}$ SOS Anesthesia and Reanimation, Ospedale del Mugello, UsI Toscana Centro, Borgo San Lorenzo, Florence, Italy. ${ }^{10}$ Department of Health Science, Section of Anesthesia and Critical Care, University of Florence, Florence, Italy. ${ }^{11}$ Department of Anesthesia and Critical Care, Azienda Ospedaliero-Universitaria Careggi, Florence, Italy. ${ }^{12}$ UOC Anesthesia, Reanimation and Intensive Care, Ospedale Bolognini, Seriate, Bergamo, Italy. ${ }^{13}$ Futura Diagnostica srl, Avellino, Italy. ${ }^{14}$ Department of Intensive Care, Université Libre de Bruxelles (ULB), Bruxelles, Belgium. ${ }^{15}$ Department of Inflammation and Immunology, Humanitas Clinical and Research Centre - IRCCS, Milan, Italy. ${ }^{16}$ Department of Surgical, Oncological and Oral Science (Di.Chir.On.S.), University of Palermo, Palermo, Italy. ${ }^{17}$ Department of Anesthesia, Intensive Care and Emergency, Policlinico Paolo Giaccone, Palermo, Italy.

Received: 14 July 2021 Accepted: 12 November 2021 Published online: 26 November 2021

\section{References}

1. Singer M, Deutschman CS, Seymour CW, Shankar-Hari M, Annane D, Bauer $M$, et al. The third international consensus definitions for sepsis and septic shock (Sepsis-3). JAMA. 2016;315:801-10.

2. Sakr Y, Jaschinski U, Wittebole X, Szakmany T, Lipman J, Ñamendys-Silva SA, et al. Sepsis in intensive care unit patients: worldwide data from the intensive care over nations audit. Open Forum Infect Dis. 2018;5:ofy313.

3. Rhodes A, Evans LE, Alhazzani W, Levy MM, Antonelli M, Ferrer R, et al. Surviving sepsis campaign: international guidelines for management of sepsis and septic shock: 2016. Intensive Care Med. 2017:43:304-77.

4. Venet F, Rimmelé T, Monneret G. Management of sepsis-induced immunosuppression. Crit Care Clin. 2018;34:97-106.

5. Angus DC, van der Poll T. Severe sepsis and septic shock. N Engl J Med. 2013:369:840-51.

6. Taccone FS, Stordeur P, De Backer D, Creteur J, Vincent J-L. Y-Globulin levels in patients with community-acquired septic shock. Shock. 2009;32:379-85.

7. Shankar-Hari M, Culshaw N, Post B, Tamayo E, Andaluz-Ojeda D, BermejoMartín JF, et al. Endogenous IgG hypogammaglobulinaemia in critically ill adults with sepsis: systematic review and meta-analysis. Intensive Care Med. 2015;41:1393-401.

8. Giamarellos-Bourboulis EJ, Tziolos N, Routsi C, Katsenos C, Tsangaris I, Pneumatikos I, et al. Improving outcomes of severe infections by multidrug-resistant pathogens with polyclonal lgM-enriched immunoglobulins. Clin Microbiol Infect. 2016;22:499-506.

9. Busani S, Roat E, Tosi M, Biagioni E, Coloretti I, Meschiari M, et al. Adjunctive immunotherapy with polyclonal lg-M enriched immunoglobulins for septic shock: from bench to bedside the rationale for a personalized treatment protocol. Front Med. 2021:8:616511.

10. Mouthon L, Kaveri SV, Spalter SH, Lacroix-Desmazes S, Lefranc C, Desai R, et al. Mechanisms of action of intravenous immune globulin in immunemediated diseases. Clin Exp Immunol. 1996;104(Suppl 1):3-9.

11. Laupland KB, Kirkpatrick AW, Delaney A. Polyclonal intravenous immunoglobulin for the treatment of severe sepsis and septic shock in critically ill adults: a systematic review and meta-analysis. Crit Care Med. 2007;35:2686-92.

12. Shankar-Hari M, Singer M, Spencer J. Can concurrent abnormalities in free light chains and immunoglobulin concentrations identify a target population for immunoglobulin trials in sepsis? Crit Care Med. 2017:45:1829-36

13. Martin-Loeches I, Muriel-Bombín A, Ferrer R, Artigas A, Sole-Violan J, Lorente $L$, et al. The protective association of endogenous immunoglobulins against sepsis mortality is restricted to patients with moderate organ failure. Ann Intensive Care. 2017:7:44.

14. Caironi P, Tognoni G, Masson S, Fumagalli R, Pesenti A, Romero M, et al. Albumin replacement in patients with severe sepsis or septic shock. N Engl J Med. 2014;370:1412-21. https://doi.org/10.1056/NEJMoa1305727.

15. Bone RC, Balk RA, Cerra FB, Dellinger RP, Fein AM, Knaus WA, et al. Definitions for sepsis and organ failure and guidelines for the use of innovative therapies in sepsis. The ACCP/SCCM Consensus Conference Committee. American College of Chest Physicians/Society of Critical Care Medicine. Chest. 1992;101:1644-55.

16. Vincent JL, Moreno R, Takala J, Willatts S, De Mendonça A, Bruining $\mathrm{H}$, et al. The SOFA (Sepsis-related Organ Failure Assessment) score to describe organ dysfunction/failure. On behalf of the Working Group on Sepsis-Related Problems of the European Society of Intensive Care Medicine. Intensive Care Med. 1996:22:707-10.

17. Masson S, Caironi P, Spanuth E, Thomae R, Panigada M, Sangiorgi G, et al. Presepsin (soluble CD14 subtype) and procalcitonin levels for mortality prediction in sepsis: data from the Albumin Italian outcome sepsis trial. Crit Care. 2014;18:R6.

18. Caironi P, Masson S, Mauri T, Bottazzi B, Leone R, Magnoli M, et al. Pentraxin 3 in patients with severe sepsis or shock: the ALBIOS trial. Eur J Clin Invest. 2017:47:73-83.

19. Vecchié A, Bonaventura A, Meessen J, Novelli D, Minetti S, Elia E, et al. PCSK9 is associated with mortality in patients with septic shock: data from the ALBIOS study. J Intern Med. 2021;289:179-92.

20. Bonaventura A, Carbone F, Vecchié A, Meessen J, Ferraris S, Beck E, et al. The role of resistin and myeloperoxidase in severe sepsis and septic shock: results from the ALBIOS trial. Eur J Clin Investig. 2020;50:e13333. 
21. Carbone F, Bonaventura A, Vecchiè A, Meessen J, Minetti S, Elia E, et al. Early osteopontin levels predict mortality in patients with septic shock. Eur J Intern Med. 2020;78:113-20.

22. Taccone FS, Caironi P, Taccone W, Latini R, Masson S, Tognoni G, et al. 1050: Gamma-globulin levels in patients with septic shock. Crit Care Med. 2013;41:A264

23. Gonzalez-Quintela A, Alende R, Gude F, Campos J, Rey J, Meijide LM, et al. Serum levels of immunoglobulins (lgG, IgA, IgM) in a general adult population and their relationship with alcohol consumption, smoking and common metabolic abnormalities. Clin Exp Immunol. 2008;151:42-50.

24. Dati F, Schumann G, Thomas L, Aguzzi F, Baudner S, Bienvenu J, et al. Consensus of a group of professional societies and diagnostic companies on guidelines for interim reference ranges for 14 proteins in serum based on the standardization against the IFCC/BCR/CAP Reference Material (CRM 470). International Federation of Clinical Chemistry. Community Bureau of Reference of the Commission of the European Communities. College of American Pathologist. Eur J Clin Chem Clin Biochem. 1996;34:517-20.

25. Puissant-Lubrano B, Peres M, Apoil P-A, Congy-Jolivet N, Roubinet F, Blancher A. Immunoglobulin IgA, IgD, lgG, IgM and IgG subclass reference values in adults. Clin Chem Lab Med. 2015;53:e359.

26. Røsjø H, Masson S, Caironi P, Stridsberg M, Magnoli M, Christensen G, et al. Prognostic value of secretoneurin in patients with severe sepsis and septic shock: data from the albumin Italian outcome sepsis study. Crit Care Med. 2018;46:e404-10.

27. Bermejo-Martín JF, Rodriguez-Fernandez A, Herrán-Monge R, AndaluzOjeda D, Muriel-Bombín A, Merino P, et al. Immunoglobulins lgG1, IgM and IgA: a synergistic team influencing survival in sepsis. J Intern Med. 2014;276:404-12.

28. Almansa R, Tamayo E, Andaluz-Ojeda D, Nogales L, Blanco J, Eiros JM, et al. The original sins of clinical trials with intravenous immunoglobulins in sepsis. Crit Care. 2015;19:90.

29. Andaluz-Ojeda D, Iglesias V, Bobillo F, Almansa R, Rico L, Gandía F, et al. Early natural killer cell counts in blood predict mortality in severe sepsis. Crit Care. 2011;15:R243.

30. Dietz S, Lautenschläger C, Müller-Werdan U, Pilz G, Fraunberger P, Päsler $M$, et al. Serum IgG levels and mortality in patients with severe sepsis and septic shock. Med Klin Intensivmed Notfmed. 2017;1 12:462-70.

31. Norrby-Teglund A, Ihendyane N, Kansal R, Basma H, Kotb M, Andersson J, et al. Relative neutralizing activity in polyspecific $\lg M$, IgA, and $\lg G$ preparations against group A streptococcal superantigens. Clin Infect Dis. 2000;31:1175-82.

32. Werdan K. Intravenous immunoglobulin for prophylaxis and therapy of sepsis. Curr Opin Crit Care. 2001;7:354-61.

33. Shankar-Hari M, Spencer J, Sewell WA, Rowan KM, Singer M. Bench-tobedside review: Immunoglobulin therapy for sepsis_-biological plausibility from a critical care perspective. Crit Care. 2012;16:206.

34. Lambris JD, Ricklin D, Geisbrecht BV. Complement evasion by human pathogens. Nat Rev Microbiol. 2008;6:132-42.

35. Giamarellos-Bourboulis EJ, Apostolidou E, Lada M, Perdios I, Gatselis NK, Tsangaris I, et al. Kinetics of circulating immunoglobulin M in sepsis: relationship with final outcome. Crit Care. 2013;17:R247.

36. Rauch PJ, Chudnovskiy A, Robbins CS, Weber GF, Etzrodt M, Hilgendorf I, et al. Innate response activator B cells protect against microbial sepsis. Science. 2012;335:597-601.

37. Venet F, Gebeile R, Bancel J, Guignant C, Poitevin-Later F, Malcus C, et al. Assessment of plasmatic immunoglobulin G, A and $M$ levels in septic shock patients. Int Immunopharmacol. 2011;11:2086-90.

38. Seymour CW, Kennedy JN, Wang S, Chang C-CH, Elliott CF, Xu Z, et al. Derivation, validation, and potential treatment implications of novel clinical phenotypes for sepsis. JAMA. 2019;321:2003-17.

39. Scicluna BP, van Vught LA, Zwinderman AH, Wiewel MA, Davenport EE, Burnham KL, et al. Classification of patients with sepsis according to blood genomic endotype: a prospective cohort study. Lancet Respir Med. 2017:5:816-26.

\section{Publisher's Note}

Springer Nature remains neutral with regard to jurisdictional claims in published maps and institutional affiliations. 